\title{
Study on the eco-control model of sustainable landscape design
}

\author{
Leichang Huang ${ }^{1, a^{*}}$, Chen Chen ${ }^{1, b}$ * \\ ${ }^{1}$ Art \& Design School, Dalian Polytechnic University, Liaoning, China \\ abaomason@163.com, b954193842@qq.com \\ ${ }^{*}$ Corresponding author
}

Keywords: Eco-control; Model; Sustainable landscape; Landscape design; Technology system. Abstract. Control can make things develop following the planning direction, and keep the change of deviation from target in the permitted limits. Ecological control (eco-control) combines ecology with cybernetics, which researches on the process of information transfer, transformation, treatment for the ecosystem and the rules of adapting and control the ecosystem.

Sustainable landscapes means contributing to human well-being and at the same time are in harmony with the natural environment. For this way, the situation, problems and development trends of modern landscape design were analyzed; the eco-control and sustainable landscape were discussed. The eco-control model for sustainable landscape design(SLD) was made with a" $1+1+2+3+1$ " construction, which means one process, one goal, two dimensions, three scales and one verification. One process refers to a complete landscape design process control of "full-life-cycle", one goal is a goal of sustainability, two dimensions mean control of theory and practice, three scales include macro, meso and micro, one verification is the evaluation system of post-design.

\section{Introduction}

With the popularization of "Sustainability" and the awakening of the human environment consciousness, Ecological design, sustainable design and sustainable landscape etc were studied in recent years both the theory and practice for the subject of landscape architecture. And there are too many sustainability-driven planning, design, construction, maintenance and so on. The concrete methods and technology, the landscape architecture elements, the various landscape sites were also studied. However, there is the need of a system model to control and lead all the actions of landscape architecture, for all above are partial or scattered and frequently the SLD is only taken as an concept without actual meaning. This paper put forward eco-control for this way from the view of ecosystem.

\section{Sustainable Landscape Design}

Landscape. Landscape is both a verb and noun in the English language, coming from Dutch firstly, the original meaning is to adapt cultural and natural processes to create new territory [1].it was also derived from the Germanic word landschaft of the early twelfth century, it initially referred to the "shape the land is in with respect to its customs, the material forms generated by those customs as law [2].

The landscape can be identified as a space unit with a regional unique element, subject and historical evolution process, which reflects the fixed structure and dynamic ecosystem with typical variability [3]. Landscape design is defined as the art of creating or enhancing outdoor features with the incorporation of plants, pavement, constructed features, landforms, and water [4].Landscape design is the design taking human as an ecosystem.

Sustainable Landscape. Sustainability is seen as a way to reintegrate technological structures with the environment in a more positive manner. There are many definitions of sustainability. The most popularly used definition of sustainable development is "that meets the needs of the present without compromising the ability of future Generations to meet the grown needs". And it was proposed for the consideration of that global population had surpassed its ecological carrying capacity. That is trying to 
establish a balance between the use and preservation of nature's potentials and resources [5].And then it popularized in various fields.

Sustainable landscape was proposed as a term in Declaration of United Nations Conference on Human Environment [6]. There are varying definitions of sustainable landscape. In 1988, the Council of Educators in Landscape Architecture defined sustainable landscapes is "contribute to human well-being and at the same time are in harmony with the natural environment" [7]. SLD is the regenerative design based on the natural system self-renew capability [8]. Sustainable landscape planning is a very forward-looking activity to improve, restore and create landscape [9].

In a word, a sustainable landscape should be good to economic, ecology, and social culture in function, only the various human activities will not destroy and harmony with nature and resources, is the sustainability able to be realized [10]. It is also a balance between human and nature. Eco-control takes this as its aim.

\section{Eco-Control Model}

Control. It is one of the most basic concepts of cybernetics, with the meaning of regulation, manipulation, control, management, command. Control is a process, an organized system adjusts itself according to internal and external changes of various conditions, overcomes the system uncertainty, to keep the system in or to achieve a particular state. Control is an action with some purpose keeping the system moving toward a certain direction by active control. Control can make things develop follow the planning direction, and keep the change of deviation from target in the permitted limits [11].

Eco-Control. There are few reports for the real eco-control in SLD. The actual meaning of eco-control is mainly in insects, microorganisms and plants population [12]. Now, it is partial only studying some concrete node of ecosystem without combining related subjects such as LA, planning etc. Eco-control is a subject that researches on the process of information transfer, transformation, treatment for the ecosystem and the rules of adapting and control the ecosystem [13]. which combines ecology with cybernetics, planning, LA, adapted to various ecosystems. The basic attributes are purpose, boundary, controllability, integrity, dynamic and operability. It takes human and nature as a whole ecosystem for a sustainable landscape.

Eco-Control Model of SLD. Based on the theories of ecology, LA, cybernetics and others related subjects, the eco-control of SLD, is to utilize eco-control technology of a control system, by the transformation and feedback of information, to make the landscape ecosystem run according to the ideal predesigned program in order to achieve a sustainable landscape ecosystem. It isn't the traditional partial methods for some problems but for the whole LA ecosystem as a tragedy for SLD.

Model Construction. Based on the attribute of sustainable landscape design and the eco-control The eco-control model for sustainable landscape design was made with a" $1+1+2+3+1$ " (Fig 1)construction, which means one process, one goal, two sides, three scales and one feedback. One process refers to a complete landscape design process control of "full-life-cycle", one goal is a goal of sustainable landscape ecosystem, two sides mean control of theory and practice, three scales include macro, meso and micro, one feedback is the evaluation system of post-design.

Model Relationships. The eco-control theory mainly controls the whole ideas of SLD, it includes the" three" scales of macro-meso-micro, in macro scale, there are "target" control theory guiding the direction, the whole planning control and the total quantity control which according to the human being needs and the nature's capacity, it also is to control the cost of all. The process management control theory in meso scale is to adjust the relationships among the various design nodes. In micro scale, the role of eco-control theory is related to the concrete design. 
The techniques for eco-control refer to the integration of technology, mainly in three sides: landscape elements of vegatation, water and terrain, road and facilities, the functional space design technology and the application technology of materials. And all the techniques are all responsible for SLD based on a view of ecology.

\section{A virtuous recycle}

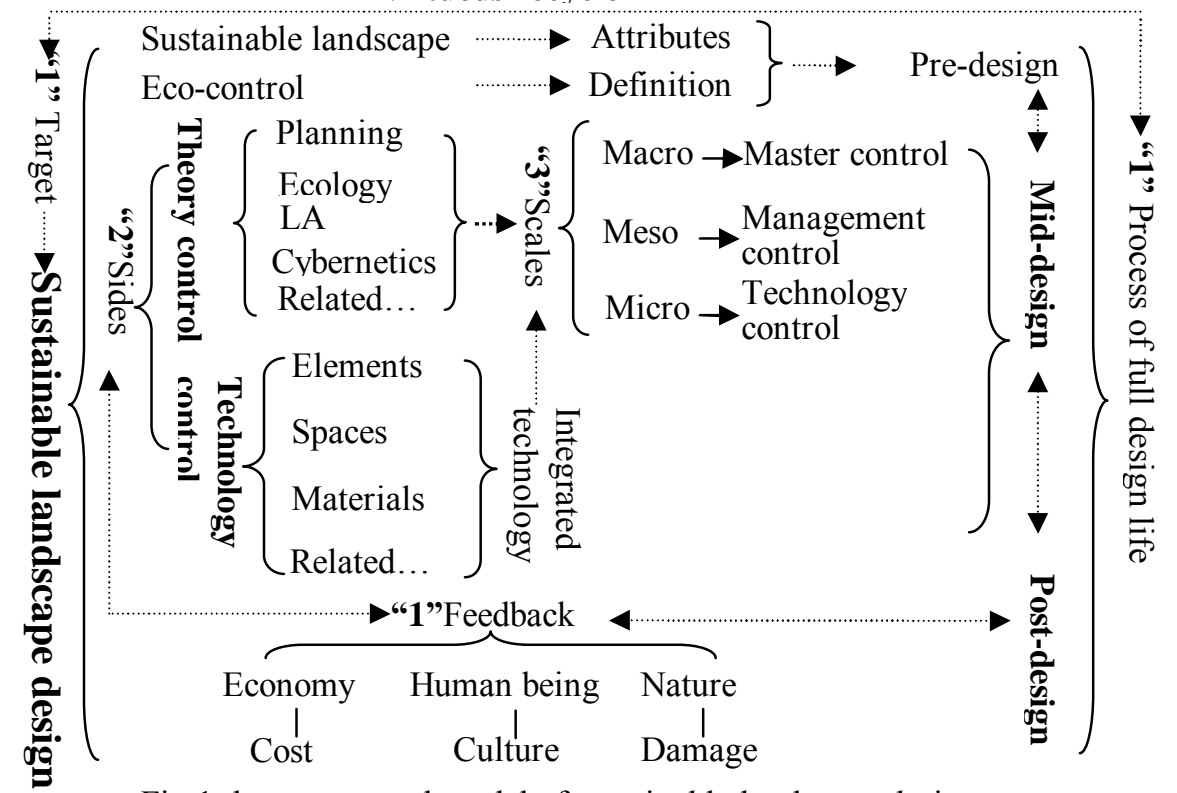

Fig 1 the eco-control model of sustainable landscape design

Feedback control. As to the feedback, it also researches on the theory \& technology, three scales and the target, testing the progress corresponding to the ideal direction. It can play an important role in the next circulation of the full-life-cycle. It is an evaluation of the SLD process in fact.

\section{Conclusions}

Eco-Control Is Not Omnipotent. Sustainable landscape is an ideal state, eco-control is a whole control for an ecosystem, and it refers to a direction but can not resolve all questions just as all other models. The eco-control is a direction and systemic idea, it cannot resolve all problems whether your interest is mainly in landscape, or mainly in sustainability, whether it is mainly as a landscape theoretician or practitioner, or even in another disciplinary area [14]. It must be a balance between human and nature, human must control their action. It is the duty of all the world people; we must obey it without exception.

Landscape design and construction are unlikely to resolve this question completely. With coordinated effort, however, the landscape professions can and must be part of the resolution [15].

Eco-Control Is an Open and Dynamic System. Sustainability should be seen as a direction, rather than a concrete goal [16].There are no fixed immutable ecological control techniques and models, which is relative, with the progress of science and society and the development and change: some disturbance will not need to control and there will be some new interference and the need to control. Of course the eco-control model is an open system. The new theory and technique will come in with the development of society, economy and culture and technology.

Of course, sustainable landscape design is not a fixed invariable concept, it is an open system, the sustainability of landscape is developing with the continuation of the time and the progress of science and technology, which is a spiral rising circulation of negation of negation. Today's sustainability or unsustainable will changes during the developing process, so the sustainability itself is a no-end system. 


\section{Acknowledgment}

The authors want to thank the anonymous reviewers for their helpful comments and suggestions. The authors are grateful to the support of the National Natural Science Foundation of China, the Grant No. 31270747 project: study on the construction of ecol-control technique system for sustainable landscape design.

\section{References}

[1] F. Steiner, Landscape ecological urbanism: origins and trajectories, J. Landscape and Urban Planning, 100 (2011) 333-337.

[2] E. David Nye, "Technologies of Landscape," Technologies of Landscape: From Reaping to Recycling, (Amherst: University of Massachusetts Press, 1999) pp.139.

[3] L .K. Christian, Our visual landscape managing the landscape under special consideration of visual aspects, J. Landscape and Urban Planning, 54 (2001) 239-254.

[4] H L.G. Hannebaum, Landscape Design: A Practical Approach. Pearson Education, Inc., Upper Saddle River, New Jersey, 2002.

[5] United Nations.Agenda21: The United Nations Programmer of Action From Rio. United Nations, New York, 1992.

[6] R. LIU, Innovative design of sustainable landscapes integrating people-oriented, history, and ecology, J. Journal of Landscape Research, 6 (2013) 25-26, 28. In Chinese.

[7] L. Robert, Jr. Thayer, The Experience of Sustainable Design, J. Landscape Journal, 8 (1989)101.

[8] J. T, Lyle, Development: Regenerative Design for Sustainable Development, John Wiley \& Sons, Inc., 1994.

[9] A. Marc. Why Landscapes of the Past are Important for the Future, J. Landscape and Urban Planning, 70 (2005) 21-34.

[10] N. Werner. Sustainable landscape use and aesthetic perception preliminary reflections on future landscape aesthetics, J. Landscape and Urban Planning, 54 (2001) 223-237.

[11] J.B. McLaughlin, F. W. Wang translation, The Application of System Approach in Urban and Regional Planning [M]. 1st ed., Building Industry Press, Beijing, 1998, In Chinese.

[12] L.H. Huang, Ecological Control Theory and Method of Corridor Construction Strong Disturbed of Island Ecosystem, [D], Fudan University, Shanghai, 2008, In Chinese.

[13] R.S. Wang, Efficient Harmony, Urban Ecological Regulation Principle, Hunan Education Press [M]. 1988, In Chinese.

[14] F. John Benson \& R. Maggie, Landscape and Sustainability, 2nd ed., 2007.

[15] W. Thompson \& Kim, Solving Sustainable Landscape Construction A Guide to Green Building Outdoors, 2nd ed., drawings by Craig D. Farnsworth, ASLA,2008.

[16] R.T.T. Forman, Land Mosaics: The Ecology of Landscapes and Regions, 2nd ed., Cambridge University Press, Cambridge, UK, 1995. 